\title{
Characterizing and targeting PDGFRA alterations in pediatric high-grade glioma
}

\author{
Carl Koschmann ${ }^{1,2}$, Daniel Zamler ${ }^{2}$, Alan MacKay ${ }^{3}$, Dan Robinson ${ }^{4}$, Yi-Mi Wu ${ }^{4}$, \\ Robert Doherty ${ }^{2}$, Bernard Marini ${ }^{5}$, Dustin Tran ${ }^{2}$, Hugh Garton ${ }^{2}$, Karin Muraszko ${ }^{2}$, \\ Patricia Robertson 6 , Marcia Leonard ${ }^{1}$, Lili Zhao7, Dale Bixby ${ }^{8}$, Luke Peterson ${ }^{8}$, \\ Sandra Camelo-Piragua ${ }^{4}$, Chris Jones ${ }^{3}$, Rajen Mody ${ }^{1}$, Pedro R. Lowenstein ${ }^{2,9}$, \\ Maria G. Castro ${ }^{2,9}$ \\ ${ }^{1}$ Department of Pediatrics, Division of Pediatric Hematology-Oncology, University of Michigan School of Medicine, Ann Arbor, \\ MI 48109, USA \\ ${ }^{2}$ Department of Neurosurgery, University of Michigan School of Medicine, Ann Arbor, MI 48109, USA \\ ${ }^{3}$ Divisions of Molecular Pathology and Cancer Therapeutics, Institute of Cancer Research, London, SM2 5NG, UK \\ ${ }^{4}$ Department of Pathology, University of Michigan School of Medicine, Ann Arbor, MI 48109, USA \\ ${ }^{5}$ Department of Pharmacology, University of Michigan School of Medicine, Ann Arbor, MI 48109, USA \\ ${ }^{6}$ Department of Pediatrics, Division of Neurology, University of Michigan School of Medicine, Ann Arbor, MI 48109, USA \\ ${ }^{7}$ Department of Biostatistics, University of Michigan School of Medicine, Ann Arbor, MI 48109, USA \\ ${ }^{8}$ Department of Internal Medicine, University of Michigan School of Medicine, Ann Arbor, MI 48109, USA \\ ${ }^{9}$ Department of Cell and Developmental Biology, University of Michigan School of Medicine, Ann Arbor, MI 48109, USA \\ Correspondence to: Maria G. Castro, email: mariacas@med.umich.edu
}

Keywords: pediatric high-grade glioma, PDGFRA amplification, PDGFRA mutation, brain tumor, tyrosine kinase inhibitor

Received: May 06, $2016 \quad$ Accepted: August 13, $2016 \quad$ Published: August 25, 2016

\section{ABSTRACT}

Pediatric high-grade glioma (HGG, WHO Grade III and IV) is a devastating brain tumor with a median survival of less than two years. PDGFRA is frequently mutated/ amplified in pediatric HGG, but the significance of this finding has not been fully characterized. We hypothesize that alterations of PDGFRA will promote distinct prognostic and treatment implications in pediatric HGG. In order to characterize the impact of PDGFR pathway alterations, we integrated genomic data from pediatric HGG patients $(n=290)$ from multiple pediatric datasets and sequencing platforms. Integration of multiple human datasets showed that PDGFRA mutation, but not amplification, was associated with older age in pediatric HGG $(P=<0.0001)$. In multivariate analysis, PDGFRA mutation was correlated with worse prognosis $(P=0.026)$, while PDGFRA amplification was not $(P=0.11)$. By Kaplan-Meier analysis, non-brainstem HGG with PDGFRA amplification carried a worse prognosis than nonbrainstem HGG without PDGFRA amplification $(P=0.021)$. There were no pediatric patients with PDGFRA-amplified HGG that survived longer than two years. Additionally, we performed paired molecular profiling (germline / tumor / primary cell culture) and targeting of an infant thalamic HGG with amplification and outlier increased expression of PDGFRA. Dasatinib inhibited proliferation most effectively. In summary, integration of the largest genomic dataset of pediatric HGG to date, allowed us to highlight that PDGFRA mutation is found in older pediatric patients and that PDGFRA amplification is prognostic in non-brainstem HGG. Future precision-medicine based clinical trials for pediatric patients with PDGFRA-altered HGG should consider the optimized delivery of dasatinib. 


\section{INTRODUCTION}

Pediatric high-grade glioma (WHO Grade III and IV) is a devastating brain tumor carrying a poor prognosis. Despite histologic similarities to adult highgrade glioma (HGG), there are important differences from tumors arising in younger patients. In particular, pediatric HGG arises in different locations, more often in midline structures such as the brainstem and thalamus. As well, pediatric high grade gliomas almost always develop de novo as high-grade lesions, as opposed to secondary GBM seen in some adult patients [1-3]. For older children with HGG, treatment is similar to adult patients, with attempt at maximal resection, followed by treatment with focal radiation, often with the addition of temozolomide. Infants are often treated with intensive multi-agent chemo with the goal of avoiding or delaying radiation [4]. These treatments are rarely curative, and $70-90 \%$ of patients with pediatric HGG will die within two years of diagnosis [2].

Recent molecular profiling of pediatric $\mathrm{HGG}$ has further highlighted important biologic differences with adult HGG. Recurrent mutations in the histone gene $H 3 F 3 A$ are seen almost exclusively in pediatric HGG, and mutations in TP53 and the histone chaperone protein ATRX are seen more frequently in pediatric HGG $[5,6]$. These histone mutations lead to epigenetic changes resulting in transcriptional changes of developmental genes, and highlight the unique pressures that may drive tumor growth in the developing brain [1]. In fact, molecular characterization of pediatric HGGs has documented key differences among different sub-populations of pediatric patients, as separated by age and location [1]. As well, treatment responses may be different, with infants possibly representing a more chemotherapy-responsive subgroup [1]. These distinctions highlight the importance of future treatments in HGG being tailored to the molecular attributes of the individual tumor of the patient.

Recent work has also documented the mutation, amplification and up-regulation of PDGFRA in a significant subset (15-39\%) of pediatric patients with HGG [2, 3]. PDGFRA is amplified less frequently in adult HGG, but has been found to carry a worse prognosis in adult anaplastic astrocytoma (WHO grade III glioma) [7]. An analysis of adult and pediatric HGGs showed that PDGFRA amplification by FISH carried a worse prognosis in adult IDHI-mutated HGG, but not in pediatric HGG [8]. Previous large scale genomic studies of pediatric HGG $[2,3]$ have not focused on the unique prognostic and treatment implication of PDGFRA alterations. In order to fully characterize the impact of PDGFRA alterations in pediatric HGG patients, we integrated genomic data from multiple datasets and sequencing platforms to create a large pediatric $\mathrm{HGG}$ genomic dataset $(\mathrm{n}=290)$.

In order to further explore the ability to target PDGFRA-amplified pediatric $\mathrm{HGG}$, we generated a novel pediatric HGG primary cell culture with confirmed PDGFRA amplification. We performed molecular characterization of the matched tumor and the primary cell culture, and describe the successful targeting of PDGFRA with clinically available receptor tyrosine kinase inhibitors. Our genomic analysis and in vitro data provide compelling evidence for the continued optimization of dasatinib delivery for pediatric HGG patients with confirmed PDGFRA alteration.

\section{RESULTS}

To assess the impact of PDGFRA alterations on survival in pediatric HGG patients, we retrieved multiple datasets of publicly available genome-wide data available in the European Genome Archive (EGA). We then integrated multiple sequencing platforms used for these datasets to produce full somatic sequence and copy number information on 290 pediatric high-grade glioma (HGG) samples (up to age 30), including 137 diffuse intrinsic pontine glioma (DIPG) and 153 nonbrainstem HGG (22 anaplastic astrocytomas (WHO grade III), 125 glioblastomas (WHO grade IV), 1 anaplastic ganglioglioma, 1 gliomatosis cerebri, and 4 high-grade glioma, not otherwise specified). Of these samples, 26 (8.9\%) carried PDGFRA mutations, 22 (7.5\%) carried PDGFRA amplifications, 6 (2.0\%) carried both PDGFRA mutation and amplification, for a total of 41 samples with PDGFRA alterations (14.1\%) (Table 1). PDGFRA amplification was not associated with TP53, FGFR1, ATRX and IDHI mutations by McNemar's test $(\mathrm{P}<$ 0.05 and kappa $<0.07$ for all comparisons)). PDGFRA mutation was not associated with TP53, FGFR1, and IDH1 mutations $(\mathrm{P}<0.001$ and kappa $<0.12$ for all comparisons). There appeared to be a slight association between PDGFRA mutation and ATRX mutation $(\mathrm{P}=0.11$ and kappa $=0.17)$.

PDGFRA mutation, but not amplification, was associated with older age in pediatric HGG (average age 14.5 years (mutated) and 9.4 years (non-mutated); $\mathrm{P}=$ $<0.0001$ ) (Figure 1A). PDGFRA alterations combined (mutation and/or amplification) were also seen in older patients (13.1 years) compared to PDGFRA wildtype pediatric HGG (9.3 years; $\mathrm{P}=0.003$ ). PDGFRA amplification was more frequently found in the brainstem; compared to PDGFRA mutation, which was more frequently hemispheric (Figure 1B).

PDGFRA amplification was associated with worse overall survival, when compared by Kaplan-Meier analysis (Figure 2A, $P=0.0058$ ). PDGFRA mutation, on the other hand, was not associated with a survival 
Table 1: Characteristics of pediatric HGGs with PDGFRA alterations

\begin{tabular}{|c|c|c|c|c|c|c|c|c|c|c|}
\hline & sample ID & sex & $\begin{array}{c}\text { PDGFRA } \\
\text { mutation }\end{array}$ & $\begin{array}{c}\text { PDGFRA } \\
\text { mutation type }\end{array}$ & $\begin{array}{l}\text { PDGFRA } \\
\text { copy number }\end{array}$ & age & diagnosis & location & $\begin{array}{c}\text { OS } \\
\text { (if known) }\end{array}$ & $\begin{array}{c}\text { status } \\
\text { (if known) }\end{array}$ \\
\hline \multirow{6}{*}{$\begin{array}{l}P D G F R A \\
\text { mutation and } \\
\text { amplification }\end{array}$} & pHGG_194 & $\mathrm{F}$ & N468S & Missense & AMP & 12.9 & AA & Hemispheric & 4.6 & DOD \\
\hline & pHGG_266 & M & Y288C & Missense & AMP & 22.7 & AA & Hemispheric & 18.0 & DOD \\
\hline & pHGG_126 & $\mathrm{F}$ & N659K & Missense & AMP & 7.6 & DIPG & Brainstem & 12.7 & DOD \\
\hline & pHGG_127 & M & T281P & Missense & AMP & 7.8 & DIPG & Brainstem & 4.4 & DOD \\
\hline & pHGG_226 & M & I843fs & IF del & AMP & 14.8 & GBM & Hemispheric & 13.0 & DOD \\
\hline & pHGG_138 & $\mathrm{F}$ & $\mathrm{A} 341 \mathrm{~T}$ & Missense & AMP & 8.7 & GBM & Midline & & \\
\hline \multirow{20}{*}{$\begin{array}{l}\text { PDGFRA } \\
\text { mutation } \\
\text { alone }\end{array}$} & pHGG_64 & $\mathrm{F}$ & $543 \mathrm{fs}$ & IF ins & $\mathrm{NC}$ & 5.3 & DIPG & Brainstem & 8.7 & DOD \\
\hline & pHGG_66 & $\mathrm{F}$ & A529fs & IF ins & $\mathrm{NC}$ & 5.3 & DIPG & Brainstem & & \\
\hline & pHGG_175 & $\mathrm{F}$ & $\mathrm{A} 341 \mathrm{~T}$ & Missense & $\mathrm{NC}$ & 11.0 & DIPG & Brainstem & & \\
\hline & pHGG_191 & $\mathrm{F}$ & N659K & Missense & $\mathrm{NC}$ & 12.5 & DIPG & Brainstem & 9.6 & DOD \\
\hline & pHGG_58 & M & K385I & Missense & $\mathrm{NC}$ & 5.0 & GBM & Hemispheric & 6.0 & DOD \\
\hline & pHGG_102 & M & Y288C & Missense & $\mathrm{NC}$ & 6.5 & GBM & Hemispheric & 9.9 & DOD \\
\hline & pHGG_168 & $\mathrm{F}$ & $384 \mathrm{fs}$ & FS & $\mathrm{NC}$ & 10.9 & GBM & Hemispheric & & \\
\hline & pHGG_224 & $\mathrm{F}$ & E311fs & FS & $\mathrm{NC}$ & 14.4 & GBM & Hemispheric & 16.5 & DOD \\
\hline & pHGG_238 & $\mathrm{F}$ & D583fs & IF del & $\mathrm{NC}$ & 15.8 & GBM & Hemispheric & 12.9 & DOD \\
\hline & pHGG_243 & M & R491fs & IF ins & $\mathrm{NC}$ & 16.7 & GBM & Hemispheric & 9.2 & DOD \\
\hline & pHGG_254 & $\mathrm{F}$ & $\mathrm{C} 235 \mathrm{Y}$ & Missense & $\mathrm{NC}$ & 19.0 & GBM & Hemispheric & 21.0 & DOD \\
\hline & pHGG_259 & $\mathrm{F}$ & K385M & Missense & $\mathrm{NC}$ & 20.0 & GBM & Hemispheric & & \\
\hline & pHGG_262 & $\mathrm{F}$ & V561A & Missense & $\mathrm{NC}$ & 21.0 & GBM & Hemispheric & 25.0 & alive \\
\hline & pHGG_268 & $\mathrm{F}$ & Y288C & Missense & $\mathrm{NC}$ & 24.0 & GBM & Hemispheric & & \\
\hline & pHGG_269 & M & D576G & Missense & $\mathrm{NC}$ & 25.0 & GBM & Hemispheric & 0.1 & DOD \\
\hline & pHGG_272 & M & $\mathrm{C} 235 \mathrm{Y}$ & Missense & $\mathrm{NC}$ & 27.0 & GBM & Hemispheric & 15.0 & DOD \\
\hline & pHGG_277 & M & V336fs & IF del & $\mathrm{NC}$ & 30.0 & GBM & Hemispheric & 27.0 & alive \\
\hline & pHGG_51 & M & N659K & Missense & $\mathrm{NC}$ & 4.7 & GBM & Midline & 6.7 & DOD \\
\hline & pHGG_183 & $\mathrm{F}$ & D842fs & NonFS indel & $\mathrm{NC}$ & 12.0 & GBM & Midline & 8.0 & DOD \\
\hline & pHGG_248 & $\mathrm{M}$ & Y555C & Missense & $\mathrm{NC}$ & 17.2 & GBM & Midline & 11.5 & DOD \\
\hline \multirow{12}{*}{$\begin{array}{l}P D G F R A \\
\text { amplification } \\
\text { alone }\end{array}$} & pHGG_12 & $\mathrm{M}$ & & & AMP & 1.8 & DIPG & Brainstem & 20.9 & DOD \\
\hline & pHGG_46 & $\mathrm{F}$ & & & AMP & 4.5 & DIPG & Brainstem & & \\
\hline & pHGG_95 & M & & & AMP & 6.1 & DIPG & Brainstem & 14.4 & DOD \\
\hline & pHGG_99 & $\mathrm{F}$ & & & AMP & 6.4 & DIPG & Brainstem & 5.5 & DOD \\
\hline & pHGG_112 & M & & & AMP & 7.0 & DIPG & Brainstem & & \\
\hline & pHGG_119 & M & & & AMP & 7.2 & DIPG & Brainstem & 6.0 & DOD \\
\hline & pHGG_125 & M & & & AMP & 7.6 & DIPG & Brainstem & 2.8 & DOD \\
\hline & pHGG_158 & $\mathrm{F}$ & & & AMP & 10.0 & DIPG & Brainstem & 10.0 & DOD \\
\hline & pHGG_165 & M & & & AMP & 10.6 & DIPG & Brainstem & 10.2 & DOD \\
\hline & pHGG_227 & $\mathrm{F}$ & & & AMP & 15.0 & DIPG & Brainstem & 13.4 & DOD \\
\hline & pHGG_236 & $\mathrm{M}$ & & & AMP & 15.6 & DIPG & Brainstem & 1.2 & DOD \\
\hline & pHGG_178 & $\mathrm{M}$ & & & AMP & 11.5 & GBM & Hemispheric & 5.0 & alive \\
\hline
\end{tabular}

(Continued) 


\begin{tabular}{lllllcllcccc}
\hline sample ID & sex & $\begin{array}{c}\text { PDGFRA } \\
\text { mutation }\end{array}$ & $\begin{array}{c}\text { PDGFRA } \\
\text { mutation type }\end{array}$ & $\begin{array}{c}\text { PDGFRA } \\
\text { copy number }\end{array}$ & age & diagnosis & location & $\begin{array}{c}\text { OS } \\
\text { (if known) }\end{array}$ (if known) \\
\hline pHGG_242 & F & & & AMP & 16.6 & GBM & Hemispheric & 12.0 & DOD \\
pHGG_252 & M & & AMP & 17.8 & GBM & Hemispheric & 8.9 & DOD \\
& pHGG_265 & M & & AMP & 22.7 & GBM & Hemispheric & 11.9 & DOD \\
\hline
\end{tabular}

IF del = in-frame deletion; FS = frameshift; IF ins = in-frame insertion; NonFS indel = non frameshift insertion/deletion; $\mathrm{AMP}=$ amplified; $\mathrm{NC}=$ normal copy number; $\mathrm{AA}=$ anaplastic astrocytoma; $\mathrm{DIPG}=$ diffuse intrinsic pontine glioma; $\mathrm{GBM}=$ glioblastoma; $\mathrm{OS}=$ overall survival; $\mathrm{DOD}=$ died of disease.

A

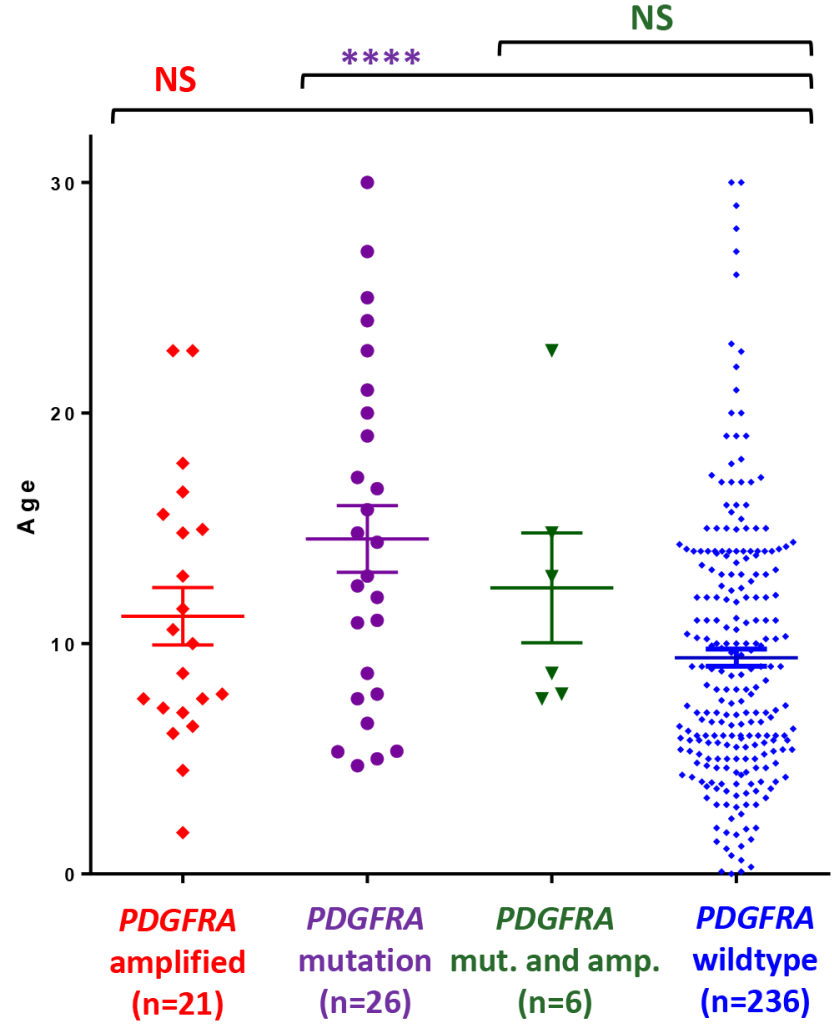

B

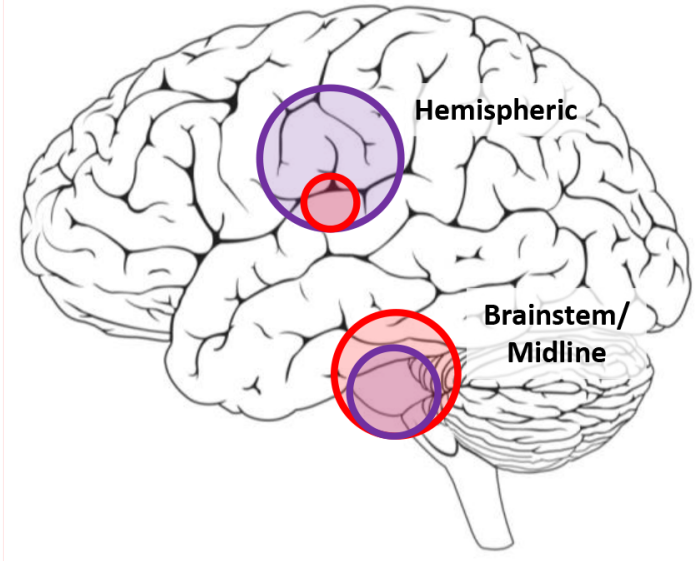

\begin{tabular}{|c|c|c|}
\hline Pediatric HGG & $\begin{array}{c}\text { PDGFRA } \\
\text { amplified }\end{array}$ & $\begin{array}{c}\text { PDGFRA } \\
\text { mutated }\end{array}$ \\
\hline DIPG & $13(61 \%)$ & $6(23 \%)$ \\
\hline AA (Midline) & $0(0 \%)$ & $0(0 \%)$ \\
\hline AA (Hemispheric) & $2(9 \%)$ & $2(7 \%)$ \\
\hline GBM (Midline) & $1(5 \%)$ & $4(15 \%)$ \\
\hline GBM (Hemispheric) & $5(23 \%)$ & $14(53 \%)$ \\
\hline Total & 21 & 26 \\
\hline
\end{tabular}

Figure 1: PDGFRA mutation is seen in older pediatric HGG patients. A. Individual data points represent individual patients in pediatric HGG dataset ( $\mathrm{n}=290$ ), with lines representing mean and standard error of measurement (SEM). PDGFRA mutation (purple) was associated with older age in pediatric HGG compared to non-mutated PDGFRA (blue). Comparison was made using an unpaired t-test $(* * * *=\mathrm{P}<0.0001 ; * * *=\mathrm{P}<0.001$, NS $=\mathrm{P}>0.05)$. B. Location of PDGFRA mutation (purple circles) and PDGFRA amplification (red circles) is represented by circle size that is proportional to frequency in pediatric HGG patients. $* * * *=\mathrm{P}<0.0001 ; * * *=\mathrm{P}<0.001 ; \mathrm{NS}=$ $\mathrm{P}>0.05 ; \mathrm{AA}=$ anaplastic astrocytoma; $\mathrm{GBM}=$ glioblastoma.

difference (Figure 2B, $\mathrm{P}=0.26$ ). When separated by anatomical location and Kaplan-Meier analysis, PDGFRA amplification remained associated with worse prognosis in non-brainstem HGG (Figure $2 \mathrm{C}, \mathrm{P}=0.026$ ), but not brainstem HGG (DIPG) (Figure 2D, $\mathrm{P}=0.26$ ). However, when using a multivariate analysis and adjusting for age and location, PDGFRA mutation was correlated with worse prognosis $(\mathrm{P}=0.026)$, while $P D G F R A$ amplification was not $(\mathrm{P}=0.11)$.

In order to further explore the biology of PDGFRAamplified pediatric $\mathrm{HGG}$, we performed molecular characterization and primary cell culture generation of pediatric patients presenting to the University of Michigan with HGG. A two-year old patient presented to 
the University of Michigan with a new infiltrative intracranial mass centered in the region of the left thalamus with extension into the left basal ganglia (Figure 3A). The tumor was partially resected and revealed a hypercellular spindle neoplasm, with prominent mitotic activity, without definite microvascular proliferation or necrosis. The tumor was strongly immuno-reactive for GFAP (Figure 3B), SMARCB/INI-1 was preserved, and the tumor was negative for chromogranin, synaptophysin, Cam 5.2, EMA, desmin, myogenin, neurofilament and mutantspecific IDH1 (R132H). Proliferation index (Ki-67) was markedly elevated (about 60\%) (Figure 3B). These histologic findings (and the molecular profiling below) were consistent with a high-grade glioma (grade III/IV); but lack of microvascular proliferation or necrosis in examined tissue did not allow us to differentiate between WHO grade III or IV glioma.

Integrative clinical sequencing revealed somatic tumor gene amplifications and outlier increased expression of PDGFRA, MYC, PVT1, CHIC2, RBPJ, FGF2, ING4, and ZNF384 (Figure 4A-4B and full sequencing details in Supplementary Figure S1). The tumor showed no H3F3A or TP53 mutations, which are frequently seen in this patient population [3].

In order to generate the primary tumor cell culture (UMPED05), fresh tumor tissue was collected at the time of resection, non-enzymatically dissociated, filtered and maintained in serum-containing media without any additional growth factor supplementation. The tumor cells grew adherently in vitro. We performed molecular

\section{A}

Pediatric HGG (all sites) by PDGFRA amplification

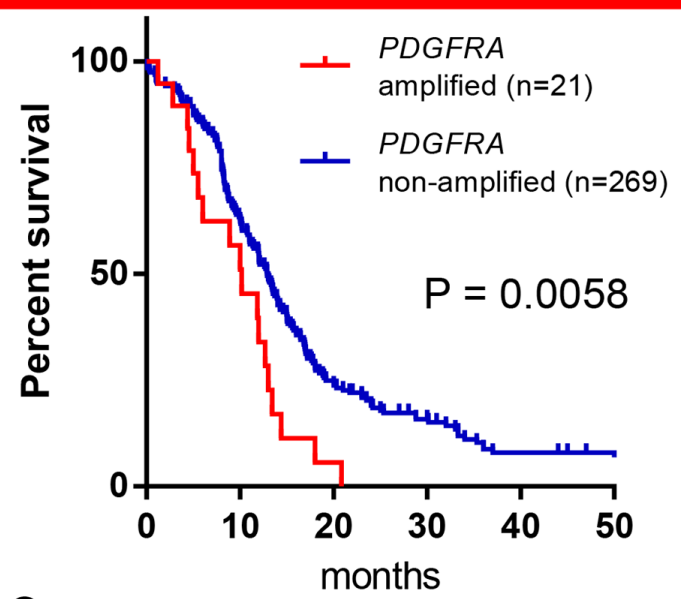

C

Non-brainstem HGG by PDGFRA amplification

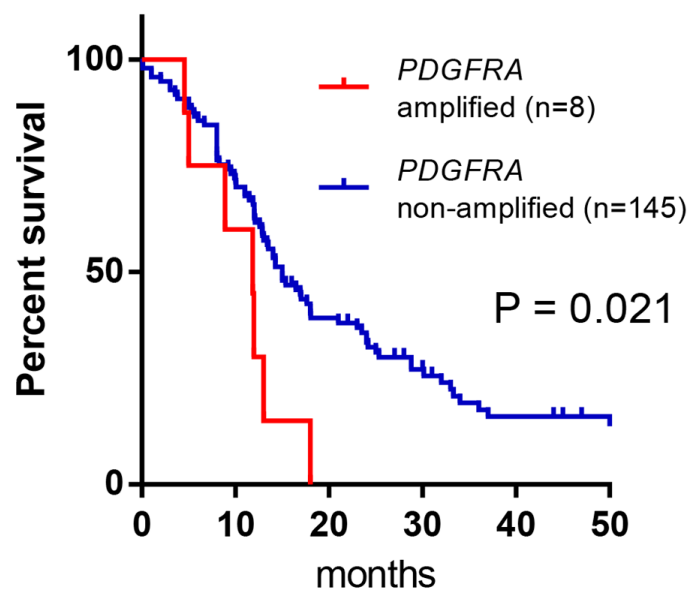

B

Pediatric HGG (all sites) by PDGFRA mutation

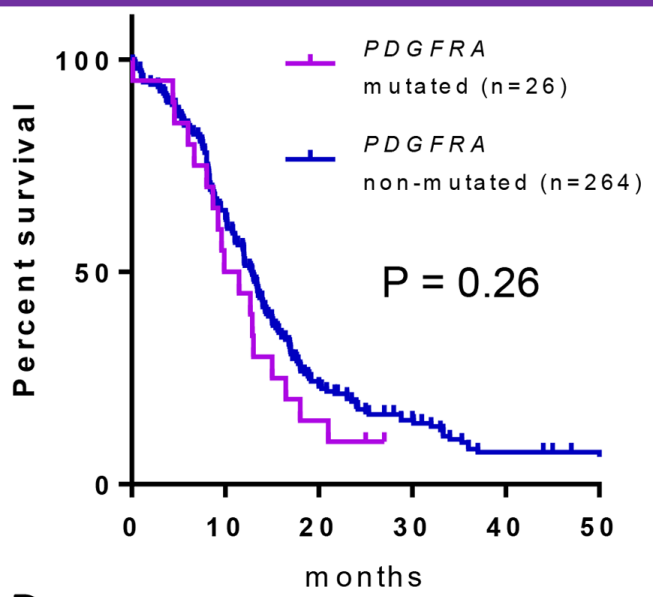

D

\section{Brainstem HGG (DIPG) by PDGFRA amplification}

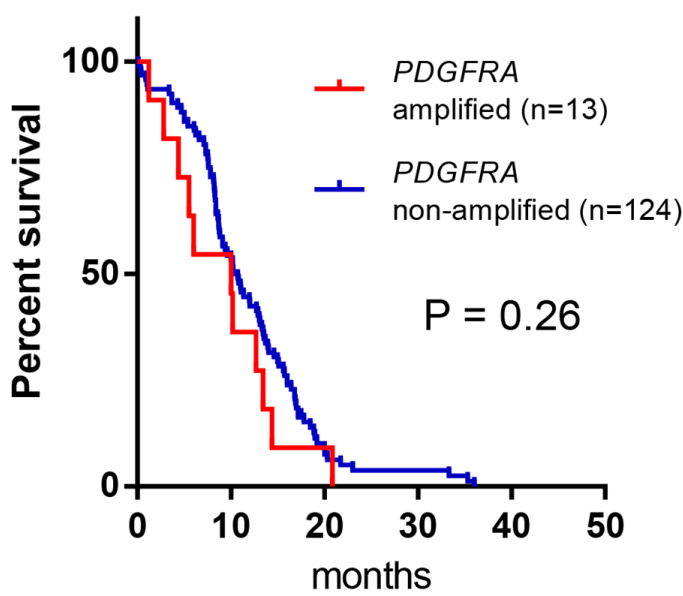

Figure 2: PDGFRA amplification is associated with worse prognosis in pediatric HGG. A. Kaplan-Meier analysis of overall survival of 290 pediatric high-grade glioma patients from multiple integrated sequencing datasets, as divided by PDGFRA amplification status, with $P D G F R A$-amplified patients (red) having significantly reduced overall survival. B. No difference was seen in survival in pediatric HGG patients by PDGFRA mutational status. PDGFRA amplification was associated with worse prognosis in non-brainstem HGG C. but not brainstem HGG (DIPG) D. 

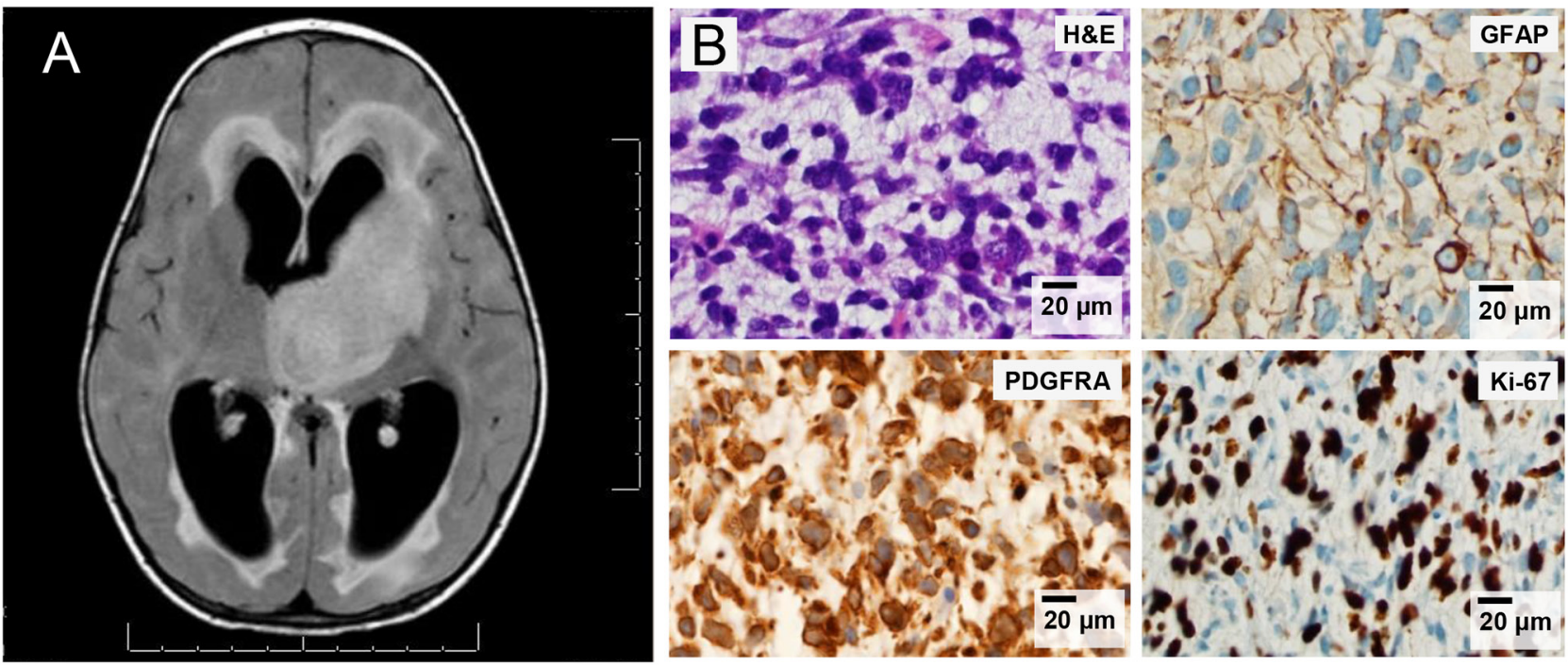

Figure 3: Clinical detail for infant with thalamic pediatric HGG (UMPED05). A. FLAIR-imaging of left thalamic tumor in two year old patient at diagnosis. B. Tumorhistology (Hematoxilin and Eosin) shows hypercellular glial (GFAP-positive) tumor with diffuse PDGFRA-positivity and elevated proliferation index (Ki67).

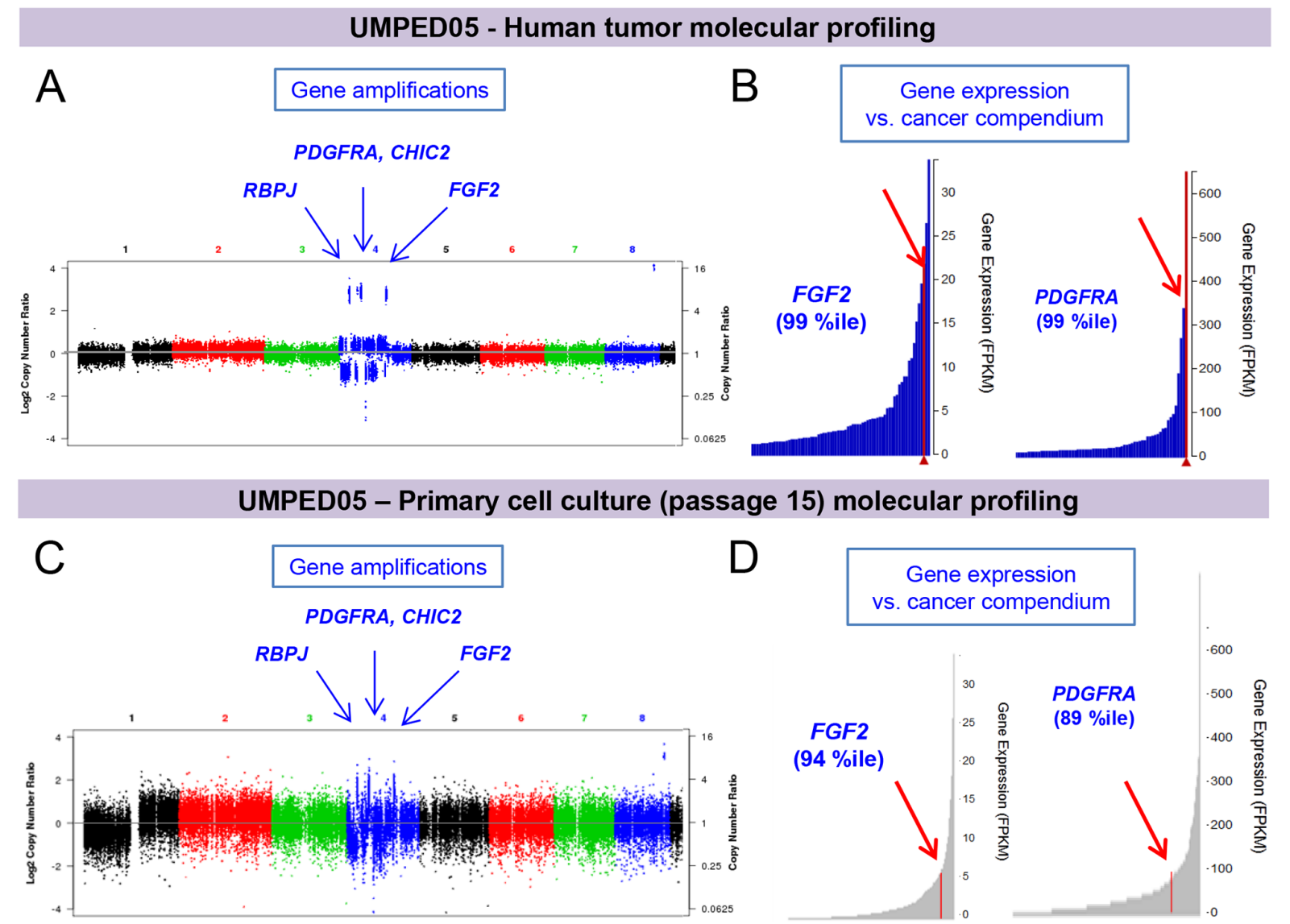

Figure 4: Integrative clinical sequencing results of paired tumor and primary cell culture from UMPED05. A. Molecular profiling shows focal gene amplifications on chromosomes 1-8, including chromosome 4 (PDGFRA, CHIC2, RBPJ and FGF2), and (not shown) chromosome 8 (MYC and PVT1) and 12 (ING4, and ZNF384); and B. increased expression of FGF2 and PDGFRA, as seen in a plot of tumor transcriptome (RNA) sequencing data. C-D. Molecular profiling of the cells in culture at passage 15 demonstrates retention of the key somatic events seen in the original human tumor, including amplification and outlier expression of PDGFRA and FGF2. 
profiling (exome and transcriptome sequencing) of the cells in culture at passage 15, and found them to continue to display the key somatic events seen in the original human tumor, including amplification and outlier expression of PDGFRA and FGF2 (among others) (Figure 4C-4D). The only genetic differences that we could establish in the cultured cells were sub-clonal gains of point mutations in MLL3 ( 10\%), which are of unclear significance (Supplementary Figure S2).

After three passages, UMPED05 cells were treated with clinically-available tyrosine kinase inhibitors known to target PDGFR signaling, including imatinib (Novartis), nilotinib (Novartis), and dasatinib (BristolMyers Squibb). Of the tyrosine kinase inhibitors used, dasatinib inhibited proliferation most effectively, with the lowest $\mathrm{IC}_{50}$ (Figure 5A). Our cell proliferation assay correlated with cell counting assessment of viability for all agents (data not shown). We additionally treated UMPED05 cells with CCNU and temozolomide, which are used clinically in pediatric HGG patients. Temozolomide showed no significant potency $\left(\mathrm{IC}_{50}=\right.$ $\sim 1 \mathrm{mM}$ ), while CCNU showed moderate potency ( $\mathrm{IC}_{50}$ $=100 \mathrm{uM}$ ) (Figure 5A). Suberanilohydroxamic acid (SAHA) is a histone deacetylase (HDAC) inhibitor which has been studied in recent clinical trials of pediatric and adult high-grade glioma patients $[9,10]$. Treatment of UMPED05 with SAHA showed moderate potency, with an $\mathrm{IC}_{50}$ of $10 \mu \mathrm{M}$ (Figure 5A). In order to determine whether reduction in proliferation was specific to reduction in PDGFRA expression and/or FGF2 expression (UMPED05 harbors amplifications in both), we performed in vitro targeting of UMPED05 cells with multiple siRNAs. Reduction in proliferation was observed in transfection with one siPDGFRA, but not with two siFGF2s (Supplementary Figure S3).

Finally, we explored whether the treatment with dasatinib was specific to UMPED05. We selected another established adherent (non-growth factor supplemented) pediatric GBM cell culture (KNS42) which has been sequenced and found to have no growth factor receptor amplifications (COSMIC ID 907282) [11]. KNS42 was less sensitive to dasatinib, with a $\sim 400$ fold higher $\mathrm{IC}_{50}$ than UMPED05 (Figure 5B). We were unable to acquire additional HGG cell cultures with PDGFRA alterations, which limited our ability to confirm that the potency of dasatinib was generalizable to all glioma cells with PDGFRA up-regulation.

\section{DISCUSSION}

Previous large scale genomic characterizations of pediatric HGG have shown that PDGFRA is a frequent tumor driver $[2,3]$. However, the prognostic and treatment implications of PDGFRA alteration in pediatric $\mathrm{HGG}$ have not been fully characterized. By integrating genomic data from multiple datasets, we were able to and generate the largest pediatric HGG genomic dataset to date, with standardized somatic event calling and an adequate sample size for sub-group analysis.

In our dataset, the number of total number of pediatric patients with PDGFRA amplification or mutation $(14.1 \%)$ was near the lower end of the range of previously published datasets [2, 3]. We report that PDGFRA mutation is prognostic in pediatric HGG. Previous largescale characterizations of PDGFRA alterations in glioma have not reported this, either because they have focused on adults patients [7] or amplification (by FISH) alone [8]. PDGFRA amplification was not prognostic by multivariate analysis, which may, in part, be explained by the overlap between amplification and location (brainstem), which is highly prognostic. Non-brainstem pediatric $\mathrm{HGG}$ with PDGFRA amplification carried a worse prognosis than non-brainstem HGG without PDGFRA amplification, by Kaplan-Meier analysis $(\mathrm{P}=0.021)$. There were no patients with PDGFRA amplification with reported survival beyond two years in our datasets.

Since the genomic data used in our analysis does not include treatment data, it is possible that differences in outcomes among molecular sub-groups in our analyses may have been influenced by additional treatment factors. For, example, the small number of patients $(n=8)$ with PDGFRA-amplified non-brainstem HGG may have received different therapy than the larger non-amplified group. Nevertheless, prognostic difference between PDGFRA mutation and amplification in pediatric $\mathrm{HGG}$ highlights the importance of genomic characterization at the DNA level for future risk-stratification, as both may lead to changes at the RNA and protein level.

The poor prognosis seen in all sub-groups of pediatric $\mathrm{HGG}$, but particularly DIPG, further highlights the clear need to improve therapies for this patient population. Our method of integrative sequencing and co-generation of a primary cell culture at the time of diagnosis of a pediatric HGG offers a feasible approach to optimize and validate personalized molecular targets.

Dasatinib is a promising agent for pediatric $\mathrm{HGG}$ with PDGF pathway alterations (Figure 5C). Dasatinib is an orally bioavailable tyrosine kinase inhibitor with $\sim 60$ fold greater inhibition of PDGFR signaling than earlier generation TKIs, such as imatinib [12]. While it was originally developed for targeting the BCR-ABL gene fusion, it exhibits nanomolar range activity against PDGFRA in leukemia [13]. In the previous pediatric Phase I trial using dasatinib in patients with leukemia and solid tumors, it was found to be well tolerated [14].

Dasatinib displays moderately favorable characteristics for blood-brain barrier penetration and has demonstrated efficacy in adult patients with CNS metastases of CML [15], where CSF concentrations ( $3 \mathrm{nMol} / \mathrm{L}$ and 20 $\mathrm{nMol} / \mathrm{L}$ ) were near the $\mathrm{IC}_{50}$ we obtained for UMPED05 $(50 \mathrm{nMol} / \mathrm{L})$ and previously published tumor cell cultures (4 nMol/L) [12]. Importantly, recent work has shown that 
dasatinib (or other TKI) delivery to brain tumor parenchyma may be further improved by strategies to inhibit active efflux proteins (P-glycoprotein (P-gp) and breast cancer resistance protein (Bcrp1)). Recent pre-clinical strategies have included co-administration of TKIs with agents that inhibit these proteins, including mammalian target of rapamycin (mTOR) inhibitors [16], or elacridar [17].

When used as a single agent in recurrent adult GBM, dasatinib did not show efficacy [18]. This failure in adult patients may in part be related to the increased frequency of multiple tumor driving mutations in adult HGG [19]. Pediatric tumors, including $\mathrm{HGG}$, are frequently driven by fewer somatic mutations than adult tumors, and may therefore be more ideal candidates for molecularly targeted agents [20]. Additionally, optimization of future precision-medicine based therapies for HGG (adult or pediatric) may benefit from combining targeted agents for multiple key altered pathways.

For this patient (UMPED05), attempt for treatment with dasatinib was not considered until she had failed all

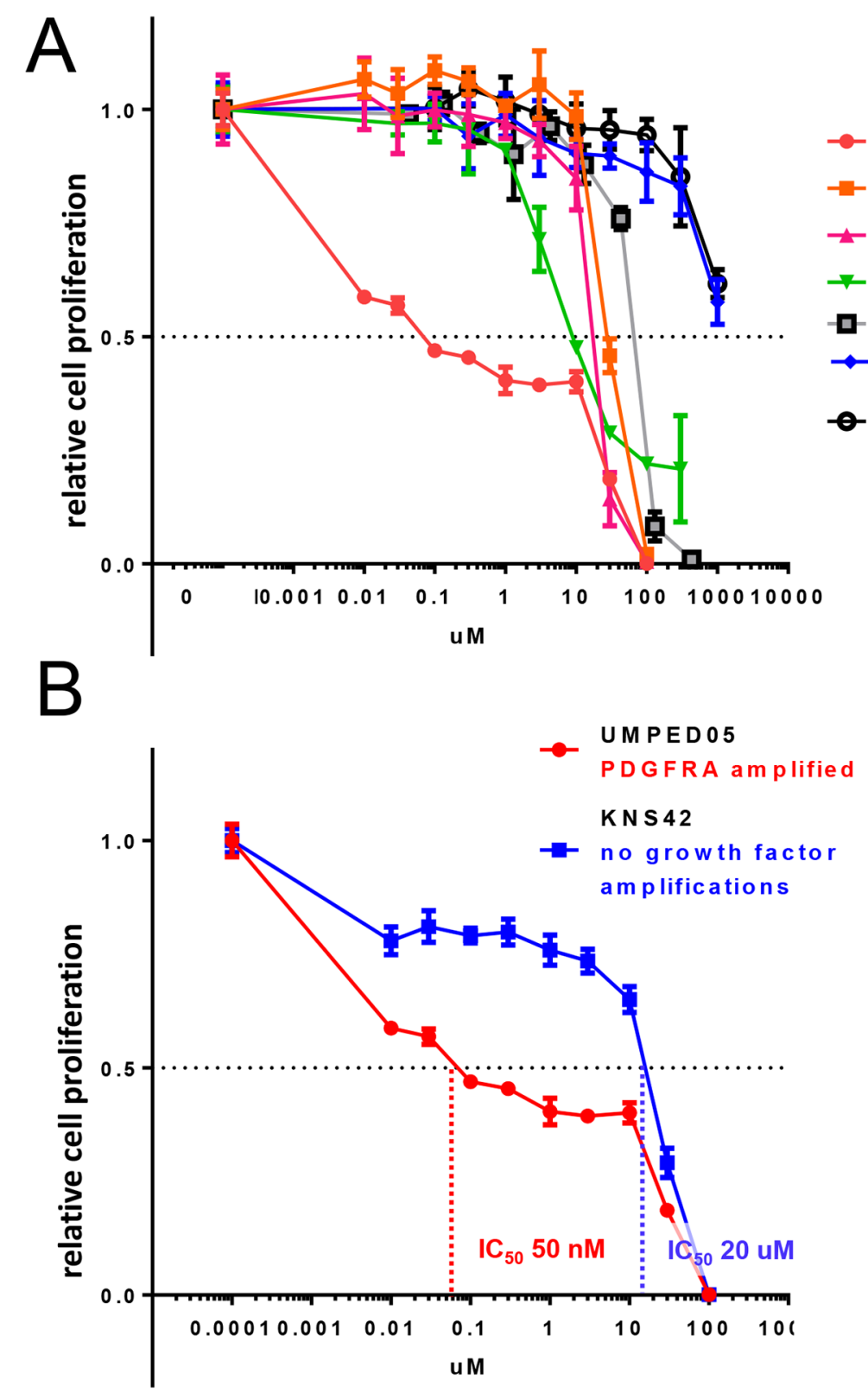

U M PED 05

PDGFRA amplified

- dasatinib (IC $50-50 \mathrm{nM}$ )

- im atinib (IC $50-40$ u M)

- nilotinib (IC $50-20 \mathrm{uM}$ )

$\because$ SAHA (IC $50-10 \mathrm{uM}$ )

- $\operatorname{CCNU}\left(\mathrm{IC}_{50}-100 \mathrm{uM}\right.$ )

$\sim$ temo (IC $\left.{ }_{50} \sim 1 \mathrm{~m} \mathrm{M}\right)$

$D M S O\left(\mathrm{IC}_{50} \sim 1 \mathrm{~m} \mathrm{M}\right)$

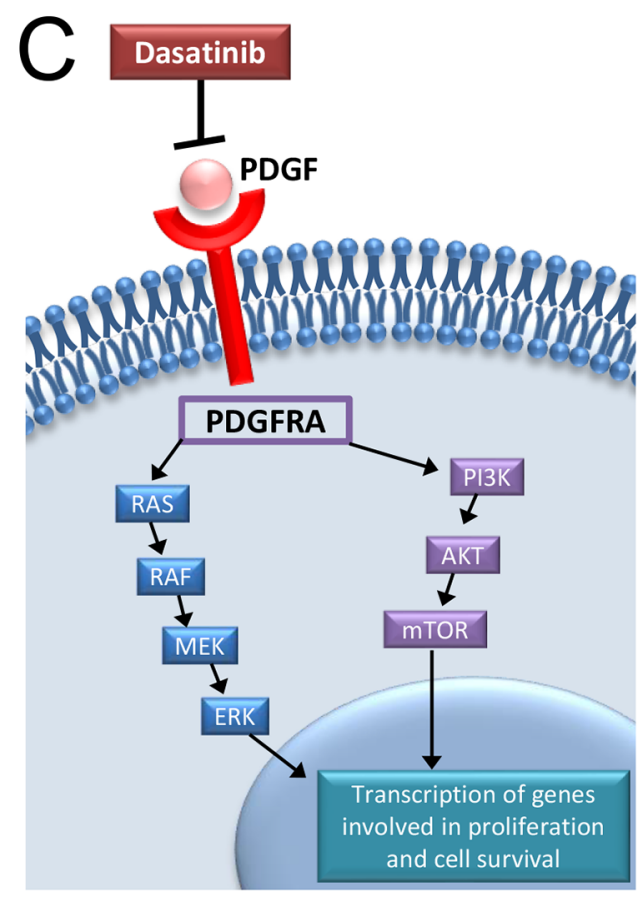

Figure 5: Treatment of UMPED05 with multiple chemotherapeutic agents reveals unique sensitivity to dasatinib. A. Dose-response curves were generated by adding chemotherapeutic agents at doses ranging from $0.01-1000 \mu \mathrm{M}$. After 48 hours of treatment, ATP levels were measured (relative light units (RLU)) in triplicate using Cell-Titer Glo and plotted versus drug concentration. Cells were most sensitive to dasatinib treatment (in red). B. UMPED05 is more sensitive (lower $\mathrm{IC}_{50}$ ) than KNS42, a pediatric GBM cell line without growth factor amplifications. C. Schematic representing impact of dasatinib on PDGFRA pathway. 
standard therapy (surgery and multi-agent chemotherapy designed for infants with brain tumors). Unfortunately, the patient passed away shortly after the tumor progressed, and so we were unable to determine whether the patient's tumor would have responded to dasatinib clinically. Future precision-medicine based clinical trials for pediatric patients with HGG could consider up-front therapy with dasatinib for tumors with PDGFRA alterations.

In summary, we describe the integration of multiple pediatric $\mathrm{HGG}$ genomic datasets which allowed us to highlight that $P D G F R A$ mutation is found in older pediatric patients and that $P D G F R A$ amplification is prognostic in non-brainstem HGG. As well, we highlight the characterization and targeting of a novel pediatric HGG primary cell culture with PDGFRA amplification. Our work highlights the importance of genomic characterization of pediatric HGG for risk stratification and targeted therapies. Hopefully, this will be a step further in providing improved outcomes for this patient population.

\section{MATERIALS AND METHODS}

\section{Human pediatric high-grade glioma dataset mutation analysis}

Human pediatric glioma mutation assessment was performed using publically-available matched tumor/ non-tumor genome sequencing datasets, all of which were accessed through the European Genome-phenome Archive (EGA), accession numbers EGAS00001000192,[3, 21] EGAS00001000572 [22], EGAS00001000575 [23] EGAS0000100072[24], and additional pediatric highgrade glioma samples (deposited at EGAS00001001436). Pediatric and young adult patients were included (age $<30$ years old at time of diagnosis), and both brainstem and non-brainstem samples were included. Somatic variants were called in alignments, using GATK version 2 and annotated for consequences with the Ensembl variant effect predictor, as previously described [6]. Copy number variations were assigned using exon level log ratios of sequence coverage in tumor/normal pairs for all known genes. Log ratios were segmented using circular binary segmentation in the DNA copy package in R version 3.1.3 and contiguous copy number aberrations were assigned using thresholds for gains/losses and amplifications/ deletions based upon the average genome wide median absolute deviation in each dataset. Survival analysis was performed using Kaplan-Meier analysis (GraphPad Software, Inc.) Cox proportional hazards model was used to assess effects of somatic mutation factors, age and location on the overall survival. Kappa statistics were calculated to quantify the association between somatic alterations and McNemar's test was used to test the association. Significance is determined if $\mathrm{P}<0.05$. All analyses were conducted using SAS (version 9.4, SAS Institute, Cary, NC).

\section{Human tumor immunohistochemistry}

Immunohistochemistry was performed in formalinfixed, paraffin embedded tissue, 5 um sections, using antibodies against GFAP (1:3200, Dako Corporation), PDGFRA (1:100, Santa Cruz) and Ki67 (Clone 30-9 prediluted, Ventana Medical Systems).

\section{Molecular profiling human tumor tissue}

Consent was obtained from the family to molecularly characterize the tumor (UMPED05) through the PEDS-MIONCOSEQ Integrative Sequencing Study, as well as to create a primary cell culture for further in vitro characterization and testing. Paired whole exome tumor DNA, cultured tumor cell DNA (passage 15), and germline DNA; and tumor and cultured tumor transcriptome sequencing was performed. In brief, nucleic acid preparation and high-throughput sequencing were performed using standard protocols in our Clinical Laboratory Improvement Amendments (CLIA) compliant sequencing lab. Paired-end whole exome libraries from tumor and matched normal DNAs captured by SureSelect Human All Exon V4 (Agilent, Santa Clara, CA), and transcriptome libraries from either poly-adenylated tumor RNA (PolyA+ transcriptome), or from total RNA captured by human all exon probes (capture transcriptome; Agilent, Santa Clara, CA) were prepared and sequenced using the Illumina HiSeq 2500 (Illumina Inc, San Diego, CA). Aligned exome and transcriptome sequencing reads were analyzed to detect putative somatic mutations, insertions/ deletions (indels), copy-number alterations, gene fusions, and gene expression as described previously [25, 26].

\section{Cell culture}

Human tumor primary cell culture UMPED05 was generated by harvesting tumor cells at the time of tumor resection by members of the University of Michigan Neuro-Oncology Translational Laboratory. The tumor tissue was transferred on ice and immediately dissociated using non-enzymatic cell dissociation buffer (Gibco, 13151-014), filtered and maintained in adherent cell line media [DMEM/F12 with L-Glutamine (Gibco, 11320033)], 10\% Fetal Bovine Serum (Gibco, 10437-077), Antibiotic-Antimycotic (Gibco 15240-062), and Normocin (Invivogen).

The pediatric GBM cell culture KNS42 [27] was generously provided by Dr. Alan Meeker at Johns Hopkins University, and maintained in above conditions to grow adherently.

\section{Treatment of cells with chemotherapy and siRNA}

For high-grade glioma primary cell cultures treatment assays, cells were passaged and plated in a 96- 
well plate at 2,000 cells/well in triplicate per condition. At 24 hours, cells were treated with chemotherapeutic agents, siRNA, or control, and assessed for viability 72 hours later using the Cell Titer Glo Assay (Promega) [28]. ON-TARGET PLUS siRNAs (GE Healthcare Dharmacon) against PDGFRA (J003162-11; J003162-12) and FGF2 (J006695-05; J006695-06) were used according to manufacturer's protocol. Chemotherapy was administered at a range of concentrations $(0.01-1000 \mu \mathrm{M})$ based on previously published data: temozolomide (Sigma), CCNU (Sigma), Suberanilohydroxamic acid (SAHA), imatinib (Novartis), nilotinib (Novartis), and dasatinib (BristolMyers Squibb). Dose response curves were plotted using GraphPad Software, and $\mathrm{IC}_{50}$ was calculated as the dose at which there was a $50 \%$ reduction in cell proliferation form untreated cells.

\section{ACKNOWLEDGMENTS}

The authors would like to thank M. Dahlgren for superb administrative support.

\section{CONFLICTS OF INTEREST}

The authors have no conflicts of interest to declare.

\section{GRANT SUPPORT}

This work was supported by National Institutes of Health/National Institute of Neurological Disorders \& Stroke (NIH/NINDS) Grants R37-NS094804, R01-NS074387, R01-NS057711, R21-NS091555, and BioInterfaces Institute, University of Michigan U042841 to M.G.C.; NIH/ NINDS Grants R01-NS054193, R01-NS061107, R01NS082311, and R21-NS084275 to P.R.L.; the Department of Neurosurgery, University of Michigan School of Medicine; the Michigan Institute for Clinical and Health Research, NIH UL1-TR000433; University of Michigan Cancer Biology Training Grant, NIH/NCI (National Cancer Institute) T32CA009676; University of Michigan Training in Clinical and Basic Neuroscience, NIH/NINDS T32-NS007222; the University of Michigan Medical Scientist Training Program, NIH/NIGMS (National Institute of General Medicine Sciences) T32-GM007863, and very generous support from Phil F. Jenkins. PEDS-MIONCOSEQ study was supported by NIH Clinical Sequencing Exploratory Research (CSER) Award NIH 1UM1HG006508 (PI: Arul M. Chinnaiyan, MD, Ph.D.) C.K. was supported by the St. Baldrick's Foundation Fellowship, the Alex's Lemonade Stand Foundation / Northwestern Mutual Young Investigator Grant, and the University of Michigan Department of Pediatrics Janette Ferrantino Investigator Award. C.J. and A.M. acknowledge NHS funding to the NIHR Biomedical Research Centre at The Royal Marsden and the ICR, and the INSTINCT network funded by The Brain Tumour Charity, Great Ormond Street Children's Charity and Children with Cancer UK.

\section{REFERENCES}

1. Jones $\mathrm{C}$ and Baker SJ. Unique genetic and epigenetic mechanisms driving paediatric diffuse high-grade glioma. Nature reviews Cancer. 2014; 14.

2. Paugh BS, Qu C, Jones C, Liu Z, Adamowicz-Brice M, Zhang J, Bax DA, Coyle B, Barrow J, Hargrave D, Lowe J, Gajjar A, Zhao W, et al. Integrated molecular genetic profiling of pediatric high-grade gliomas reveals key differences with the adult disease. Journal of clinical oncology. 2010; 28:3061-3068.

3. Wu G, Diaz AK, Paugh BS, Rankin SL, Ju B, Li Y, Zhu X, Qu C, Chen X, Zhang J, Easton J, Edmonson M, Ma X, et al. The genomic landscape of diffuse intrinsic pontine glioma and pediatric non-brainstem high-grade glioma. Nature genetics. 2014; 46:444-450.

4. Geyer JR, Sposto R, Jennings M, Boyett JM, Axtell RA, Breiger D, Broxson E, Donahue B, Finlay JL, Goldwein JW, Heier LA, Johnson D, Mazewski C, et al. Multiagent chemotherapy and deferred radiotherapy in infants with malignant brain tumors: a report from the Children's Cancer Group. Journal of clinical oncology. 2005; 23:7621-7631.

5. Schwartzentruber J, Korshunov A, Liu XY, Jones DT, Pfaff E, Jacob K, Sturm D, Fontebasso AM, Quang DA, Tonjes M, Hovestadt V, Albrecht S, Kool M, et al. Driver mutations in histone H3.3 and chromatin remodelling genes in paediatric glioblastoma. Nature. 2012; 482:226-231.

6. Koschmann C, Calinescu AA, Nunez FJ, Mackay A, Fazal-Salom J, Thomas D, Mendez F, Kamran N, Dzaman M, Mulpuri L, Krasinkiewicz J, Doherty R, Lemons $\mathrm{R}$, et al. ATRX loss promotes tumor growth and impairs nonhomologous end joining DNA repair in glioma. Science translational medicine. 2016; 8:328ra328.

7. Alentorn A, Marie Y, Carpentier C, Boisselier B, Giry M, Labussiere M, Mokhtari K, Hoang-Xuan K, Sanson M, Delattre JY and Idbaih A. Prevalence, clinico-pathological value, and co-occurrence of PDGFRA abnormalities in diffuse gliomas. Neuro Oncol. 2012; 14:1393-1403.

8. Phillips JJ, Aranda D, Ellison DW, Judkins AR, Croul SE, Brat DJ, Ligon KL, Horbinski C, Venneti S, Zadeh G, Santi M, Zhou S, Appin CL, et al. PDGFRA amplification is common in pediatric and adult high-grade astrocytomas and identifies a poor prognostic group in IDH1 mutant glioblastoma. Brain pathology. 2013; 23:565-573.

9. Keshelava N, Houghton PJ, Morton CL, Lock RB, Carol H, Keir ST, Maris JM, Reynolds CP, Gorlick R, Kolb EA, $\mathrm{Wu} \mathrm{J}$ and Smith MA. Initial testing (stage 1) of vorinostat (SAHA) by the pediatric preclinical testing program. Pediatric blood \& cancer. 2009; 53:505-508.

10. Galanis E, Jaeckle KA, Maurer MJ, Reid JM, Ames MM, Hardwick JS, Reilly JF, Loboda A, Nebozhyn M, Fantin VR, Richon VM, Scheithauer B, Giannini C, et al. Phase II trial of vorinostat in recurrent glioblastoma multiforme: a north central cancer treatment group study. Journal of clinical oncology. 2009; 27:2052-2058. 
11. Forbes SA, Bindal N, Bamford S, Cole C, Kok CY, Beare D, Jia M, Shepherd R, Leung K, Menzies A, Teague JW, Campbell PJ, Stratton MR and Futreal PA. COSMIC: mining complete cancer genomes in the Catalogue of Somatic Mutations in Cancer. Nucleic acids research. 2011; 39(Database issue):D945-950.

12. Chen Z, Lee FY, Bhalla KN and Wu J. Potent inhibition of platelet-derived growth factor-induced responses in vascular smooth muscle cells by BMS-354825 (dasatinib). Molecular pharmacology. 2006; 69:1527-1533.

13. Bixby DL and Talpaz M. Efficacy of various doses and schedules of second-generation tyrosine kinase inhibitors. Clinical lymphoma \& myeloma. 2008; 8 Suppl 3:S95-S106.

14. Aplenc R, Blaney SM, Strauss LC, Balis FM, Shusterman $\mathrm{S}$, Ingle AM, Agrawal S, Sun J, Wright JJ and Adamson PC. Pediatric phase I trial and pharmacokinetic study of dasatinib: a report from the children's oncology group phase I consortium. Journal of clinical oncology. 2011; 29:839-844.

15. Porkka K, Koskenvesa P, Lundan T, Rimpilainen J, Mustjoki S, Smykla R, Wild R, Luo R, Arnan M, Brethon B, Eccersley L, Hjorth-Hansen H, Hoglund M, et al. Dasatinib crosses the blood-brain barrier and is an efficient therapy for central nervous system Philadelphia chromosome-positive leukemia. Blood. 2008; 112:1005-1012.

16. Minocha M, Khurana V, Qin B, Pal D and Mitra AK. Co-administration strategy to enhance brain accumulation of vandetanib by modulating P-glycoprotein (P-gp/Abcb1) and breast cancer resistance protein (Bcrp1/Abcg2) mediated efflux with m-TOR inhibitors. International journal of pharmaceutics. 2012; 434:306-314.

17. Mittapalli RK, Chung AH, Parrish KE, Crabtree D, Halvorson $\mathrm{KG}, \mathrm{Hu} \mathrm{G}$, Elmquist $\mathrm{WF}$ and Becher OJ. ABCG2 and ABCB1 limit the efficacy of dasatinib in a PDGF-B driven brainstem glioma model. Molecular cancer therapeutics. 2016.

18. Lassman AB, Pugh SL, Gilbert MR, Aldape KD, Geinoz S, Beumer JH, Christner SM, Komaki R, DeAngelis LM, Gaur R, Youssef E, Wagner H, Won M and Mehta MP. Phase 2 trial of dasatinib in target-selected patients with recurrent glioblastoma (RTOG 0627). Neuro Oncol. 2015; 17:992-998.

19. Brennan CW, Verhaak RG, McKenna A, Campos B, Noushmehr H, Salama SR, Zheng S, Chakravarty D, Sanborn JZ, Berman SH, Beroukhim R, Bernard B, Wu CJ, et al. The somatic genomic landscape of glioblastoma. Cell. 2013; 155:462-477.
20. Janeway KA, Place AE, Kieran MW and Harris MH. Future of clinical genomics in pediatric oncology. Journal of clinical oncology. 2013; 31:1893-1903.

21. Wu G, Broniscer A, McEachron TA, Lu C, Paugh BS, Becksfort J, Qu C, Ding L, Huether R, Parker M, Zhang J, Gajjar A, Dyer MA, et al. Somatic histone H3 alterations in pediatric diffuse intrinsic pontine gliomas and non-brainstem glioblastomas. Nature genetics. 2012; 44:251-253.

22. Taylor KR, Mackay A, Truffaux N, Butterfield YS, Morozova O, Philippe C, Castel D, Grasso CS, Vinci M, Carvalho D, Carcaboso AM, de Torres C, Cruz O, et al. Recurrent activating ACVR1 mutations in diffuse intrinsic pontine glioma. Nature genetics. 2014; 46:457-461.

23. Buczkowicz P, Hoeman C, Rakopoulos P, Pajovic S, Letourneau L, Dzamba M, Morrison A, Lewis P, Bouffet E, Bartels U, Zuccaro J, Agnihotri S, Ryall S, et al. Genomic analysis of diffuse intrinsic pontine gliomas identifies three molecular subgroups and recurrent activating ACVR1 mutations. Nature genetics. 2014; 46:451-456.

24. Fontebasso AM, Papillon-Cavanagh S, Schwartzentruber J, Nikbakht H, Gerges N, Fiset PO, Bechet D, Faury D, De Jay N, Ramkissoon LA, Corcoran A, Jones DT, Sturm D, et al. Recurrent somatic mutations in ACVR1 in pediatric midline high-grade astrocytoma. Nature genetics. 2014; 46:462-466.

25. Robinson D, Van Allen EM, Wu YM, Schultz N, Lonigro RJ, Mosquera JM, Montgomery B, Taplin ME, Pritchard CC, Attard G, Beltran H, Abida W, Bradley RK, et al. Integrative clinical genomics of advanced prostate cancer. Cell. 2015; 161:1215-1228.

26. Roychowdhury S, Iyer MK, Robinson DR, Lonigro RJ, Wu YM, Cao X, Kalyana-Sundaram S, Sam L, Balbin OA, Quist MJ, Barrette T, Everett J, Siddiqui J, et al. Personalized oncology through integrative high-throughput sequencing: a pilot study. Science translational medicine. 2011; 3:111ra121.

27. Takeshita I, Takaki T, Kuramitsu M, Nagasaka S, Machi T, Ogawa H, Egami H, Mannoji H, Fukui M and Kitamura K. Characteristics of an established human glioma cell line, KNS-42. Neurologia medico-chirurgica. 1987; 27:581-587.

28. Assi HH, Paran C, VanderVeen N, Savakus J, Doherty R, Petruzzella E, Hoeschele JD, Appelman H, Raptis L, Mikkelsen T, Lowenstein PR and Castro MG. Preclinical characterization of signal transducer and activator of transcription 3 small molecule inhibitors for primary and metastatic brain cancer therapy. The Journal of pharmacology and experimental therapeutics. 2014; 349:458-469. 